Abstract S53 Table 1 Primary and Secondary Efficacy Outcomes in the Intent-to-Treat Population (Change from Baseline at 90 Days post Final Treatment)

\begin{tabular}{|c|c|c|c|c|}
\hline & RePneu Coil Treatment $(n=23)$ & Control $(n=24)$ & Between-Group Difference in Change from Baseline & P-value \\
\hline \multicolumn{5}{|c|}{ number ( $95 \%$ confidence interval) } \\
\hline \multicolumn{5}{|c|}{ Initial Analysis $†$} \\
\hline \multicolumn{5}{|l|}{ Primary outcome } \\
\hline Mean change in SGRO & $-9.11(-14.59$ to -3.62$)$ & $1.43(-4.05$ to 6.92$)$ & $-10.54(-17.52$ to -3.56$)$ & 0.004 \\
\hline \multicolumn{5}{|l|}{ Secondary outcome } \\
\hline Mean change in TLC $(L)$ & $-0.36(-0.55$ to -0.18$)$ & $-0.25(-0.43$ to -0.07$)$ & $-0.11(-0.35$ to 0.12$)$ & 0.330 \\
\hline Mean change in RV (L) & $-0.64(0.92$ to -0.37$)$ & $-0.29(-0.57$ to -0.02$)$ & $-0.35(-0.70$ to 0.00$)$ & 0.051 \\
\hline Mean change in 6-minute Walk Test (m) & 52.98 (29.18 to 76.78) & $-17.41(-41.21$ to 6.39$)$ & 70.39 (40.10 to 100.68$)$ & $<0.001^{\ddagger}$ \\
\hline Mean percent change in $\mathrm{FEV}_{1}$ & 14.85 (7.46 to 22.23$)$ & $2.04(-5.35$ to 9.42$)$ & 12.81 (3.41 to 22.21) & $0.009^{\ddagger}$ \\
\hline
\end{tabular}

SGRQ denotes St. George's Respiratory Questionnaire, TLC total lung capacity, RV residual volume, and FEV, forced expiratory volume in 1 second.

${ }^{\dagger} P$-value determined by analysis of variance (ANOVA) with factors of treatment and site.

${ }^{\ddagger}$ Statistical significance via the Hochberg adjustment for multiplicity for secondary outcomes.

Results Significant improvements in the treatment group compared to control group were observed for the primary end point mean SGRQ $(\Delta-10.54$ points, $p=0.004)$, as well as secondary end points mean sixminute walk distance $(\Delta+70.39$ metres, $\mathrm{p}<0.001)$ and forced expiratory volume in one second $(\Delta+12.81 \%, p=0.009)$. Between group difference in change in mean residual volume did not reach significance $(\Delta-0.35$ litres, $p=0.051)$, despite a 0.64 litre reduction in the treatment group. There was a good safety profile with treatment.

Conclusions Treatment with endobronchial coils in patients with severe emphysema and hyperinflation significantly improves quality of life, exercise capacity and pulmonary function with a good safety profile. LVRCs present a novel, safe, and minimally invasive treatment option for patients with both homogenous and heterogenous emphysema, with benefits unaffected by collateral ventilation. A larger randomised controlled pivotal trial with longer follow-up is now needed. Funding shared by PneumRx and study sites.

\section{Regulating inflammation in acute lung injury}

\section{S54 THE ROLE OF PRE-RECEPTOR GLUCOCORTICOID METABOLISM IN REGULATING THE SEVERITY AND PERSISTENCE OF MURINE LUNG INJURY}

doi:10.1136/thoraxjnl-2012-202678.060

'S Lax, ${ }^{2} \mathrm{MR}$ Wilson, ${ }^{2} \mathrm{M}$ Takata, 'P Stewart, 'D Thickett. 'The University of Birmingham, Birmingham, UK; ${ }^{2}$ mperial College London, London, UK

Introduction Acute lung injury (ALI) is a major cause of respiratory failure in the critically ill patient. With a mortality rate of $40-60 \%$, $50 \%$ of survivors left with pulmonary impairment and no current licenced treatment there is a need for novel therapies. Our current research suggests that local steroid metabolism by alveolar macrophages is defective in ALI patients. As the predominant function of these cells is phagocytosis of apoptotic neutrophils during resolution of inflammation, we sort to investigate the effect of pre-receptor glucocorticoid metabolism in a murine model of ALI

Methods Using intra-tracheal instillations of LPS (50 $\mu \mathrm{g})$, we analysed the inflammatory response in wild type (WT) mice compared to those deficient in 11ß-hydroxysteroid dehydrogenase-1 (HSD-1 $\mathrm{KO})$. These mice specifically lack the enzyme which converts inactive cortisone to active cortisol. Cell infiltrates and expression of several inflammatory markers within bronchial lavage fluid (BALF), as well as tissue permeability and mouse oximetry were examined to evaluate the immune response and lung damage.

Results Intra-tracheal LPS challenge in WT mice induced a significant increase in lung permeability $(p=0.0153)$, infiltrating neutrophils $(p=0.0121)$ and recruitment of $\mathrm{CD} 11 \mathrm{c}^{+} \mathrm{CD} 11 \mathrm{~b}^{+}$monocytes $(p<0.0001)$, which was associated with significant hypoxia $(p<0.0001)$ compared to PBS-treated controls 48 hrs post-instillation.
The severity of acute neutrophilic inflammation and epithelial barrier defects as measured by lung permeability index were similar in WT and HSD-1 KO mice.

In contrast, during resolution of LPS-induced injury ( $72 \mathrm{hrs}$ postinstillation), HSD-1 KO mice had a significant accumulation of apoptotic neutrophils $(p=0.02)$ and a significant increase in $\mathrm{CD} 11 \mathrm{c}^{+} \mathrm{CD} 11 \mathrm{~b}^{+}$monocytes $(\mathrm{p}=0.0007)$ recruited into the lung compared to WT controls. Moreover, Luminex arrays revealed a significant increase in BALF levels of IL-1 $\beta(p=0.003)$ and dysregulation of IL-6, TNF $\alpha$ and CXCL1/KC during the time course.

Conclusion Our data indicate that insufficient alveolar glucocorticoid metabolism augments the duration but not initial severity of lung injury, possibly via a dysregulation of apoptotic neutrophil clearance and suggests that therapies targeting defective macrophage HSD-1 expression may have value in promoting the resolution of ALI

\section{S55 THE AMINOPEPTIDASE CD13 REGULATES HOMOTYPIC AGGREGATION OF NEUTROPHILS}

doi:10.1136/thoraxjnl-2012-202678.061

${ }^{1} \mathrm{CA}$ Fiddler, ${ }^{2} \mathrm{G}$ Murphy, ${ }^{1} \mathrm{AS}$ Cowburn, ${ }^{1} \mathrm{H}$ Parfrey, ER Chilvers'. 'Department of Medicine, University of Cambridge School of Clinical Medicine, Cambridge, UK; ${ }^{2}$ Department of Oncology, University of Cambridge, Cambridge, UK

Neutrophils are critical effector cells of the innate immune response and are recruited to sites of tissue injury in response to locally generated chemoattractants. Neutrophil recruitment is a highly regulated process involving complex interactions with the vascular endothelium and underlying tissue stroma. In addition to adhering to the endothelium, neutrophils can also self-associate, (a process known as homotypic aggregation - HA), which has been proposed to play a key role in disease states such as sepsis.

Aminopepetidase $\mathrm{N}$ or CD13 is a widely expressed membrane-bound metallopeptidase involved in the migration and invasion of cancer and endothelial cells. Neutrophils express CD13 on their cell surface, which is upregulated by TNF- $\alpha$, IL- 8 and fMLP We have shown that inhibition of aminopeptidase activity enhances the efficacy of TNF$\alpha$-induced neutrophil apoptosis (Cowburn et al. J Biol Chem 2006; 281:12458). Cross-linking anti-CD13 monoclonal antibodies (mAb) have been shown to induce HA of monocytic cells through PI3K activation (Mina-Osorio et al. J Leuk Biol 2006; 79:719). We hypothesised that CD13 may be involved in neutrophil migration and HA.

Using plasma-Percoll purified human neutrophils and a modified Boyden filtre assay we showed that IL-8 mediated neutrophil chemotaxis was not affected by either the CD13 mAb WM-15 or the aminopeptidase enzymatic inhibitor bestatin. In contrast, IL-8-mediated neutrophil migration through type 1 collagen gels was significantly impaired by WM-15 and MY7 mAbs, which both inhibit enzymatic activity and induce clustering of CD13. The non-clustering CD13 antibody WM-47 and bestatin had no effect. WM-15 and MY7 also 